\title{
Benefits of a Clinical Pharmacist in Outpatient Diabetes Disease State Management ${ }^{i}$
}

\author{
Rola A Halabi*, Wafaa Abou-Zeineddine, Margaret Ann Breakenridge \\ MedStar Washington Hospital Center, 110 Irving St. NW, Washington, DC, United States
}

Copyright@2019 by authors, all rights reserved. Authors agree that this article remains permanently open access under the terms of the Creative Commons Attribution License 4.0 International License

\begin{abstract}
Diabetes is a chronic disease state affecting millions of patients, many of which have uncontrolled diabetes and not under a physician's care. The economic and health impacts that a pharmacist can have on patients overall health is well known. As an increasing number of patients suffer from uncontrolled diabetes and its complications which makes the role of pharmacists more important than ever to provide patients with knowledge of the many therapeutic options available, appropriate self-testing techniques, and other important lifestyle modifications. The role of pharmacists has expanded to include ambulatory care in an outpatient setting. The purpose of this study is to evaluate the impact of pharmacist involvement in diabetes management in an ambulatory clinic, where there previously has been no pharmacist participation. Comparing data from a pilot study performed without pharmacist involvement, pharmacists have been able to achieve better Hgb A1C reductions than the pilot study performed in fiscal year 16 (FY16) at an average of $3.36 \pm 1.76$. Although the sample size of patients was small at only 5 , these are promising results of the impact that in depth patient education can have on improving outcomes.
\end{abstract}

Keywords Diabetes, Pharmacists, Hgb A1C, Patients

\section{Introduction}

Diabetes is a chronic disease state that has significant impact on patients. According to recent estimates, diabetes affects 30.3 million people in the United States, with approximately 7.2 million patients remaining undiagnosed. (1) The total economic cost of diabetes is estimated at \$327 billion in 2017, including $\$ 237$ billion in direct medical costs. Prescription medications accounted for $30 \%$ of medical expenditures to treat diabetes and its complications. (2,3) Because of these rising costs, pharmacists can play an integral role on a healthcare team providing patients with knowledge, resources, and support in the treatment of diabetes. There are currently a large number of therapeutic options to treat diabetes so a pharmacist's role in caring and educating patients is more vital than ever. Drug classes most commonly used to treat patients include: biguanides, sulfonylureas, thiazolidinedione, dipeptidyl peptidase-4 (DPP-4) inhibitors, glucagon-like peptide-1 (GLP-1) receptor agonists, sodium-glucose cotransporter-2 (SGLT-2) inhibitors and insulin. Pharmacists can play a major role in diabetes patient care so that better patient outcomes can be achieved and complications from diabetes can be delayed. Pharmacists have the unique ability to educate patients regarding: proper use and side effects of medications, appropriate use of self-testing supplies, proper insulin administration, screening for drug interactions, as well as recommendations for lifestyle improvements.

The purpose of this study is to evaluate the impact of pharmacist involvement in diabetes management in an ambulatory clinic, where there previously has been no pharmacist participation.

\section{Methods}

This study was performed after obtaining Institutional Review Board (IRB) approval from the hospital's Research Institute. The pharmacist joined the endocrinology team in an outpatient diabetes clinic of a large tertiary care hospital. Patients are identified and referred to be seen for follow-up in an ambulatory outpatient diabetes clinic through: the electronic medical record system [EMR], referrals from outside clinics, and referrals from inpatient admissions for diabetic complications. Eligibility criteria included patients that are age 18 years and older, have type 2 diabetes with Hgb A1C $\geq 9 \%$. Hgb A1C is a minor component of hemoglobin to which glucose is bound. The higher the glucose concentration, the higher the Hgb A1C will be. It is a means of determining the average blood glucose concentrations for the prior three months. 


\section{Visit 1}

- A needs/knowledge assessment is conducted

- Learner centered survival skills is discussed

- Initial prescriptions given and education on medications is performed

- SMBG device registration/teaching

\section{Visit 2}

- Review BG values from previous week with patient and identify areas for further improvement

- Further adjustments to medication regimens, if necessary

- Define end goals

- Provide warm handoff to Virtual Visit Center provider

\section{Week 3-12}

- Patients followed daily on Telcare ${ }^{\ominus}$ dashboard and weekly check-in with patient by providers

- Week 12: Patient is discharged back to their PCP

SMBG: Self-monitoring blood glucose; BG: blood glucose; PCP: Primary care physician

Figure 1. Diabetes Pathway Timeline of 12 Week Program

Patients are followed in an ambulatory clinic for 12 weeks by a combination of the following providers: physicians, nurse practitioners, certified diabetes educators (CDE), and pharmacists. A timeline of the 12 weeks is shown in Figure 1. Electronic medical records are utilized to collect patient information including: age, gender, diagnosis, demographics, past medical history, medication regimens, compliance, Hgb A1C, diagnostic lab results, as well as previous hospital readmissions for complications of diabetes. Provider documentation from previous encounters is reviewed to determine reasons for non-compliance, complications, and/or target A1C goals being missed.

First visits with patients were scheduled to run for two hours. During these two hours, everything is discussed from compliance to current medication regimens, typical diet and work schedules, and methods of administering injectable agents if necessary. Patient's medication regimens are adjusted, if needed, based on an algorithm set forth by the chief endocrinologist. Sometimes adjustments are made that fall outside of the algorithm in situations where patients were receiving too high insulin doses and were routinely having hypoglycemic episodes to ensure patient safety. Typically patients were asked to walk the provider through a typical day in their life from the time they wake up until the time they go to sleep. It is important to note that providers do not show judgment for patients' food choices or other habits they may have because it is important to build rapport with the patient and have an honest conversation. An important aspect of this initial interview is to gauge patient's health literacy and understanding of the importance of diet and exercise, as well as medication adherence in their diabetes management. Patient's diet changes that should be made are discussed as well as other lifestyle improvements that could be made. This initial interview is helpful to determine what possible changes may be necessary to their medication regimen. For example, sometimes patients did not eat 3 meals a day and were using meal-time insulin even if they did not eat because they were instructed to use this insulin three times per day. This initial interview allows the provider to determine the medication regimen that would be most beneficial to patients and ensure compliance. During the initial visit patients are given a blood glucose monitor (Telcare ${ }^{\circledR}$ Meter) supplied to them at no charge, which transmits their blood glucose readings in real time to members of the clinic. This allows providers to follow patients and monitor patients at any time of day. Patients are occasionally started on new medications or their insulin regimens adjusted during this initial visit and prescriptions and prior authorizations, if necessary, are sent to the patient's pharmacy.

Examples of some of the interventions that have taken place during these initial visits include patient education regarding the amount of sugar in certain beverages. It was found that patients routinely did not count the amount of sugar that they took in through beverages and were often amazed when visual representations of their sugar intake were shown to them. Sometimes the only intervention made was to have patients stop drinking all of the sugar containing products and they immediately saw a reduction in daily blood glucose levels. Additionally, explaining to 
patients what their A1C correlated to in their average blood glucose really helped to drive home the consequences of hyper and hypo-glycemic episodes. Another aspect of this initial visit is giving patients options for healthier food options that fit into their day-to-day lifestyle and work easily with their schedules.

Patients then come in during week 2 for a one-hour in person visit. During this visit, the focus is reiterating all the information given to the patient and focusing on each patient's specific needs. Typically the provider will review the last 7 days of data from the blood glucose monitor and discuss results with the patient to find areas for improvement. Any problems that patients may have had are addressed and for the next 10 weeks the provider follows patients through their preferred method of communication. The provider or a member of the team continually follows patients and if necessary adjustments are made to medication regimens throughout the ten week period. Typically there will be weekly phone calls/emails, however if there are instances of blood glucose readings that are too high or too low, the provider will follow up with the patient to find out the reasoning for those values. At the end of the twelve-week period patients take a follow-up Hgb A1C and are discharged back to their primary care physician with a letter regarding changes made to their diabetes medication regimen and education provided to the patient.

The primary outcome measures in this study are: (1) reduction of Hgb A1C and (2) reduction in hospital readmissions for diabetes management by a pharmacist compared to outcomes achieved before pharmacist involvement in order to identify the impact that can be made in improving patient care.

\section{Results}

The results compare the results of a total of 8 patients seen by a pharmacist in FY 17 and 18 compared to historical data of a pilot study performed during FY16 that did not include pharmacist involvement and enrolled 125 patients. This data is outlined in Table 1. (4)

Table 1. Data from Pilot Study FY16

\begin{tabular}{|c|c|c|c|}
\hline & $\begin{array}{c}\text { Diabetes } \\
\text { Pathway }\end{array}$ & $\begin{array}{c}\text { Control } \\
\text { Cases }\end{array}$ & P value \\
\hline $\begin{array}{c}\text { Baseline A1C } \\
\text { (\%) }\end{array}$ & 11.4 & 11.4 & 0.439 \\
\hline $\begin{array}{c}\text { 3 Month A1C } \\
\text { (\%) }\end{array}$ & 8.3 & 9.8 & $<0.001$ \\
\hline $\begin{array}{c}\text { Change in } \\
\text { A1C (\%) }\end{array}$ & -3.1 & -1.6 & $<0.001$ \\
\hline
\end{tabular}

Of the patients seen by a pharmacist in the current study, average A1C reduction was $3.36 \pm \mathbf{1 . 7 6}$ points shown in Table 2. Two patients who were seen initially by a pharmacist were lost to follow up. This is similar to the number of patients who dropped out in the pilot study and has been seen across the board.

Patients are still being followed as of October 2017 and followed to see if at the 6 month mark they further improve or maintain their Hgb A1C. Patients in the study made great improvements in their diabetes management and many still have further room for improvement. It is important to note once patients are discharged back to their primary care physicians, if they are unable to keep up the progress made in the diabetes clinic, they can be referred back to the clinic for further management.

Table 3. Hgb A1C reduction of patients seen by pharmacist in study

\begin{tabular}{|c|c|c|c|}
\hline Patient & $\begin{array}{c}\text { Entry } \\
\text { Hgb A1C }\end{array}$ & $\begin{array}{c}\text { Exit } \\
\text { Hgb A1C }\end{array}$ & $\begin{array}{c}\text { Reduction of } \\
\text { Hgb A1C }\end{array}$ \\
\hline A & 12.6 & 9.9 & 2.7 \\
\hline B & 12.5 & 6.6 & 5.9 \\
\hline C & 9.0 & 7.6 & 1.4 \\
\hline D & 16.1 & 11.1 & 5 \\
\hline E & 11.4 & 9.5 & 1.9 \\
\hline F & 12.1 & 8.8 & 3.3 \\
\hline $\begin{array}{c}\text { Average } \\
\text { Reduction }\end{array}$ & 12.3 & 8.9 & $\mathbf{3 . 3 6} \pm \mathbf{1 . 7 6}$ \\
\hline
\end{tabular}

Data from this study demonstrate the impact that targeted diabetes education and medication management can have to reduce: Hgb A1C, hospital readmissions, and healthcare utilization measures. Because of the positive results seen with pharmacist involvement, it will continue as this clinic continues to grow and recruit more patients. The positive feedback received from patients and fellow providers in clinic of having a pharmacist talk to the patient shows the unique capabilities that a pharmacist has in managing a chronic disease state.

\section{Conclusions}

Diabetes is a chronic disease state that has significant impact on patients. It is truly a disease state that requires a multi-disciplinary approach to gain better control and improve patient outcomes. The purpose of this study was to show that pharmacists can have significant impact on patient's health and are integral members of the healthcare team. The outcomes in this study, although ongoing, are very promising and show the importance of pharmacists as part of the healthcare team.

Opportunities for improvement are evident as not every patient is at goal; however every patient improved their Hgb A1C and have not had hospital admissions for diabetes complications. 


\section{REFERENCES}

[1] [Internet]. Diabetes. org. 2018 [cited 6 September 2018]. Available from: http://www.diabetes.org/assets/pdfs/basics /cdc-statistics-report-2017.pdf

[2] [Internet]. Diabetes. org. 2018 [cited 6 September 2018]. Available from: http://www.diabetes.org/assets/pdfs/basics/ cdc-statistics-report-2017.pdf

[3] Statistics about Diabetes [Internet]. American Diabetes Association. 2018 [cited 6 September 2018]. Available from: http://www.diabetes.org/diabetes-basics/statistics/

[4] Magee, et al. "Late Breaking Poster." Presented at ADA Scientific Sessions 2016, New Orleans.

http://diabetes.diabetesjournals.org/content/diabetes/suppl/ 2016/06/20/65.Supplement_1.DC1/2016_ADA_LB_Abstr acts_HiRes_FINAL_5_11_16.pdf

i *The authors do not have anything to disclose 